\title{
Nonclassical Behavior in the Capacitance of a Nanojunction
}

\author{
J. G. Hou, ${ }^{1, *}$ Bing Wang, ${ }^{2,1}$ Jinlong Yang, ${ }^{1}$ X. R. Wang, ${ }^{2}$ H. Q. Wang, ${ }^{1}$ Qingshi Zhu, ${ }^{1}$ and Xudong Xiao ${ }^{2, \dagger}$ \\ ${ }^{1}$ Structure Research Lab and Lab of Bond Selective Chemistry, University of Science and Technology of China, \\ Hefei 230026, Anhui, China \\ ${ }^{2}$ Department of Physics, The Hong Kong University of Science and Technology, Clear Water Bay, Kowloon, Hong Kong, China
}

(Received 16 January 2001)

\begin{abstract}
The capacitance of a nanojunction formed by a scanning tunneling microscope (STM) tip and a two-dimensional gold cluster was measured through the single electron tunneling spectroscopy of a double-barrier tunnel junction. By decreasing the STM tip-cluster separation, it was observed that the capacitance first increases and then decreases at short separation. This characteristic clearly deviates from the classical behavior and provides evidence for potential quantum effects on the capacitance.
\end{abstract}

DOI: 10.1103/PhysRevLett.86.5321

With the rapid development in fabrication techniques, electronic devices are gradually approaching the nanometer scale, where many classical concepts and results might no longer be applicable and quantum corrections must be made. Capacitance is one of them. In addition to the geometry and dielectric constant, which are sufficient to determine the capacitance at the macroscales and microscales, the capacitance of a nanostructure can be affected by quantum effects [1]. Theoretical studies have shown the capacitance quantum corrections to come mainly from the finite density of states (DOS) of the nanosized electrodes, the finite screening length to the electron-electron interaction, and quantum tunneling [1-6]. So far, no experiment has been made to demonstrate these quantum effects, in spite of its importance in many phenomena such as Coulomb blockade (CB) and device applications. This is in strong contrast to other physical quantities, such as conductance of mesoscopic systems, which has been well studied and well understood both experimentally and theoretically in the past two decades [7]. The experimental difficulty for studying the quantum effects on the capacitance of a nanostructured system comes from the fact that there is no effective way to isolate such a system so as to make an accurate capacitance measurement. Here we report the first experimental attempt to investigate the capacitance behavior of a nanojunction formed by a scanning tunneling microscope (STM) tip and a nanosized two-dimensional (2D) metal cluster. By measuring the capacitance of this nanojunction as a function of tip-cluster separation $d$ in a double-barrier tunnel-junction (DBTJ) geometry (via the $\mathrm{CB}$ effect), we find that as $d$ decreases, the measured capacitance first increases, as would be anticipated by the classical theory. Below a critical separation $d_{c}$, however, the capacitance starts to decrease, a behavior which is clearly nonclassical. This nonmonotonic behavior is in good qualitative agreement with the quantum effects and might represent the first experimental observation in its category, although at this stage we cannot completely rule out other potential causes.

Our experiments are conducted on a nanometer-sized DBTJ formed by positioning an STM tip above a 2D
PACS numbers: 68.37.Ef, 61.46.+w, 73.23.Hk, 73.40.Cg

gold cluster which resides on top of an alkanethiol selfassembled monolayer (SAM) on $\mathrm{Au}(111)$. The currentvoltage $(I-V)$ curves for a DBTJ show CB and Coulomb staircases behavior due to the discreteness of the charge and its associated charging energy. According to the orthodox theory [8-10], the $\mathrm{CB}$ gap width $V_{\mathrm{CB}}$ (width of the zero conductance region) in the $I-V$ curve is $e / \max \left(C_{1}, C_{2}\right)$ if the residual charge is very small [10], where $C_{1}$ and $C_{2}$ are the capacitances of the clustersubstrate and tip-cluster tunnel junctions, respectively (see upper-left inset of Fig. 1). Thus, the capacitance of the nanojunction can be deduced via measuring $V_{\mathrm{CB}}$. Compared to a DBTJ formed with a three-dimensional (3D) metal cluster passivated by organic ligands or with a naked metal cluster residing on a high dielectric constant insulating film on conducting substrates, our choice to use a naked 2D Au cluster on a low dielectric constant organic film allows the following: (1) the cluster size to be measured by STM and the classical capacitance of the corresponding nanojunctions to be computed; (2) the STM tip to approach closer to the metal cluster and thus $C_{2}$ to determine the gap width $V_{\mathrm{CB}}$ (the low dielectric constant makes $C_{1}$ relatively small); (3) the dependence of $C_{2}$ on the tip-cluster separation $d$ to be studied without altering any other conditions, but simply by adjusting the STM set point tunneling current; and (4) the DOS of the cluster to be sufficiently small in order for the quantum effect of capacitance to be observable.

The sample preparation has been described in previous publications [11,12]. Briefly, we first evaporate $\sim 160 \mathrm{~nm}$ thick gold on freshly cleaved mica heated at $300{ }^{\circ} \mathrm{C}$ to form the $\mathrm{Au}(111)$ substrates. Then a monolayer of alkanethiol, $\mathrm{CH}_{3}\left(\mathrm{CH}_{2}\right)_{9} \mathrm{SH}\left(\mathrm{C}_{10}\right)$, is self-assembled on the fresh $\mathrm{Au}(111)$ in a $2 \mathrm{mM}$ ethanol solution of $\mathrm{C}_{10}$ for $48 \mathrm{~h}$. The molecules tilt away from the surface normal by $\sim 30^{\circ}$ and thus form a layer of $1.4 \mathrm{~nm}$ thick [13]. This wellordered and densely packed monolayer is a good insulator with a dielectric constant of $\varepsilon \sim 2.7$ [14]. Such a molecular monolayer with atomically flat surface provides both the supporting layer and one tunneling barrier in the DBTJ for the metal clusters. On top of the SAM, 0.2 monolayer 


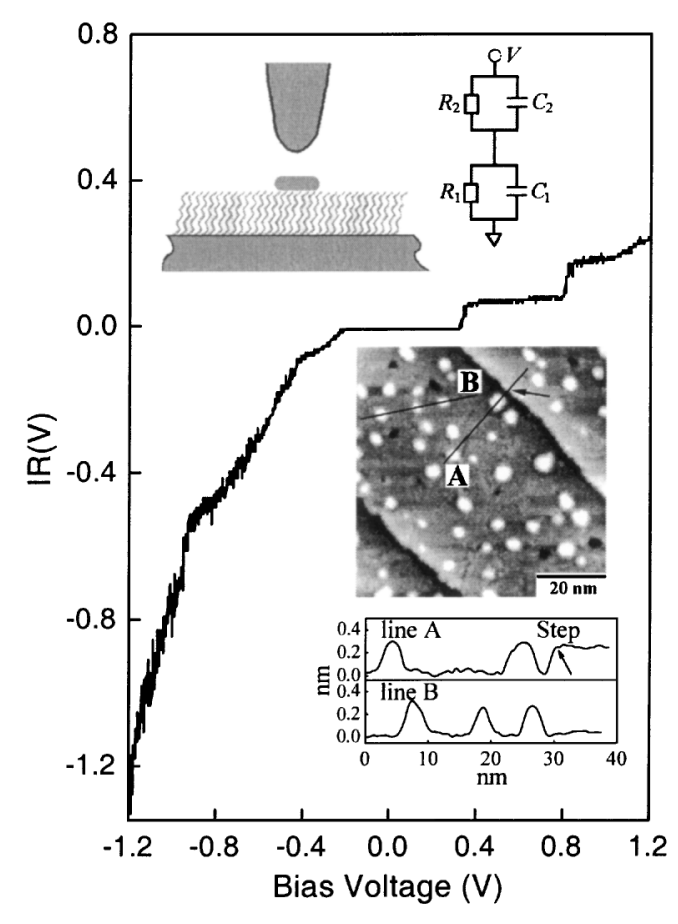

FIG. 1. A typical $I-V$ curve showing the Coulomb blockade and staircases for a 2D Au cluster of $2 \mathrm{~nm}$ size at $5 \mathrm{~K}$, with the vertical axis rescaled by the set point tunneling resistance $R=0.9 \mathrm{G} \Omega$. The upper-left inset shows the geometry of the DBTJ with an equivalent circuit diagram. The lower-right inset shows an image of the 2D Au clusters with a height of about $0.25 \mathrm{~nm}$, the same as a Au step. The rounded shape in the height profiles $A$ and $B$ of the $2 \mathrm{D}$ clusters is due to the convolution of the finite tip apex.

of gold is deposited by thermal evaporation to form $\mathrm{Au}$ clusters. The STM experiments are performed with an OMICRON low temperature UHV STM system. Before the $I-V$ curve measurement, the STM tip is first positioned above a chosen Au cluster and the tip-cluster separation is adjusted by the setting values of the bias voltage and tunneling current. Subsequently, the feedback is turned off for taking $I-V$ spectra.

As reported elsewhere [11], the Au clusters formed by this method are 2D and a fraction of them, indeed, resides on top of the SAM. As shown in the lower-right inset of Fig. 1, they have the same height as a $\operatorname{Au}(111)$ step: $\sim 0.25 \mathrm{~nm}$. The $2 \mathrm{D}$ cluster size ranges from $\sim 1$ to $\sim 10 \mathrm{~nm}$, with a typical separation of $\sim 10 \mathrm{~nm}$ between the clusters. In the main panel of Fig. 1, a typical tunneling $I-V$ curve acquired at $5 \mathrm{~K}$ from a $\sim 2 \mathrm{~nm}$ Au cluster is shown. In addition to the zero conductance regions near zero voltage, a number of current steps in both positive and negative voltage directions are observed. Although the uneven voltage spacing between the adjacent current steps is in deviation from that typically observed for 3D clusters, we find that for $2 \mathrm{D}$ Au clusters the zero conductance region is the result of $\mathrm{CB}$ [12].

In this Letter we demonstrate experimentally that the capacitance of a nanojunction as a function of the tipcluster separation is nonmonotonic. As an example, in
Fig. 2(a) are shown a series of tunneling $I-V$ curves for a $\sim 4 \mathrm{~nm}$ Au cluster measured at various electrode separation obtained by adjusting the set point tunneling current while maintaining the bias voltage. As the set point current increases (the separation $d$ decreases), the $\mathrm{CB}$ width first remains approximately unchanged, then decreases from 1 to $6 \mathrm{nA}$. Above $6 \mathrm{nA}$, the $\mathrm{CB}$ width reverses the trend to increase. Since the $\mathrm{CB}$ width is basically inversely proportional to the larger capacitance of $C_{1}$ and $C_{2}$, the above behavior indicates that the capacitance of the tip-cluster junction first increases, and then decreases as one reduces $d$. To see this behavior more quantitatively, and to avoid any ambiguity in determining the capacitances of the two junctions, we have performed a least-squares fitting to the $I-V$ curves using the orthodox theory [8-10]. In the fitting the capacitance $C_{1}$ and the resistance $R_{1}$ of the clustersubstrate junction are found to be approximately unchanged with values of $0.26 \mathrm{aF}$ and $67 \mathrm{M} \Omega$, respectively, regardless of the tip-cluster separation $d$, consistent with the assumption that the cluster-substrate junction is not affected by the tip position. The fitting value of capacitance is also close to the estimated value of $0.21 \mathrm{aF}$ using a parallel plate geometry for a $\sim 4 \mathrm{~nm}$ size plate. Keeping $C_{1}$ and $R_{1}$ constant, we can deduce $C_{2}$ and $R_{2}$ from the fitting. Because of the large nonlinear behavior at high voltages, we have to treat $R_{2}$ as a function of voltage $V$ by $R_{2}=R_{20} /\left(1+\alpha V^{2}\right)$ instead of a constant for $I$ - $V$ curves taken at high set point currents, where $\alpha$ and $R_{20}$ are constants. Reasonable agreement between the fitting and the experimental data can be achieved, in particular for $|V|<0.6 \mathrm{~V}$. The fitting results of the tip-cluster junction capacitance $C_{2}$ is plotted in Fig. 2(b), where the separation $d$ is converted from the set point current by $I=$ $I_{0} \exp (-1.025 \sqrt{\phi} d)$ to first obtain the relative values
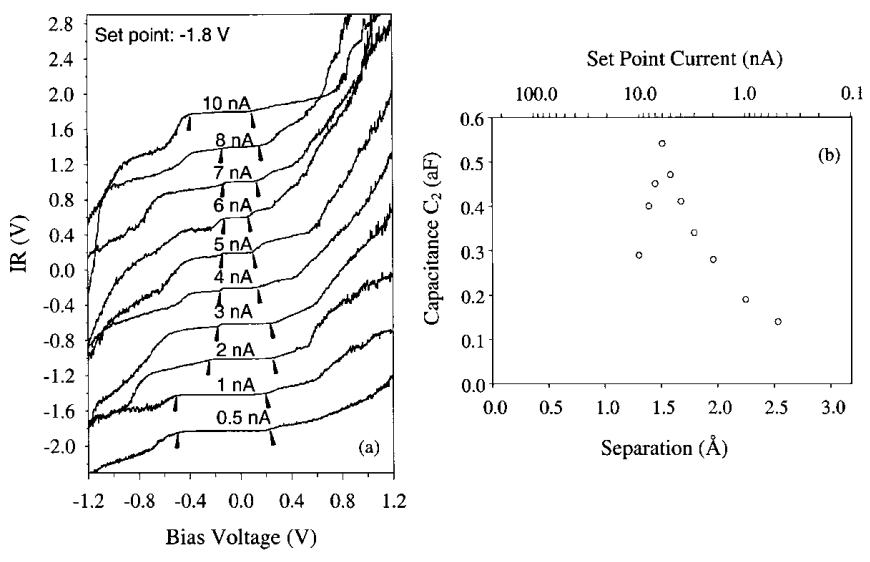

FIG. 2. (a) A series of $I-V$ curves taken at $5 \mathrm{~K}$ for a $4 \mathrm{~nm}$ $\mathrm{Au}$ cluster at different set point tunneling current, where the vertical axis is rescaled by the set point tunneling resistance $R=3.6,1.8,0.9,0.6,0.45,0.36,0.3,0.26,0.22,0.18 \mathrm{G} \Omega$ for set point tunneling current $0.5,1,2,3,4,5,6,7,8$, and $10 \mathrm{nA}$, respectively. The $\mathrm{CB}$ width is indicated by arrows for each $I-V$ curve. The curves are shifted vertically for clarity. (b) The deduced capacitance $C_{2}$ of tip-cluster nanojunction as a function of tip-cluster separation $d$. 
[15], for which the work function is taken as $\phi \sim 5 \mathrm{eV}$ for gold. The absolute value of $d$ is determined by the condition $C_{1} \sim C_{2}$ at $I \sim 1 \mathrm{nA}$ above which the CB gap starts to change substantially. A full classical calculation [12] for the given geometry with a parabolic tip of a radius $10 \mathrm{~nm}$ gives $d=2.3 \AA$ at this point, assuming that any other corrections to $C_{1}$ and $C_{2}$ are comparable. As $d$ decreases from 2.5 to $1.5 \AA, C_{2}$ increases by as much as $\sim 3$ times. As $d$ further decreases from 1.5 to $1.2 \AA$, however, $C_{2}$ decreases by a factor of $\sim 2$. Although our fitting may not give the precise values of $C_{2}$ as the fitting quality is somewhat poor in the high voltage range and the orthodox theory may not work well for 2D Au clusters by treating the cluster as metallic in the thickness direction [12], we believe the qualitative features of $C_{2}$ as a function of $d$ would not be altered even if a better theory is used. Thus, our measured capacitance of the tip-cluster nanojunction is clearly a nonmonotonic function of the separation $d$.

The physics of the observed behavior of the CB gap width is now clear. At small set point tunneling current, or large $d, V_{\mathrm{CB}}$ remains approximately unchanged because $C_{1}$ is larger than $C_{2}$ and determines the CB gap. When $d$ is adjusted small enough, $C_{2}$ becomes larger than $C_{1}$ and controls the CB gap. As one continues to decrease $d$, the CB gap width then manifests the behavior of $C_{2}$ which increases first and then starts to decrease below a critical separation $d_{c}$. This is why the CB gap width first decreases as the set point current increases from 1 to $6 \mathrm{nA}$, and then increases as the set point current further increases from 6 to $10 \mathrm{nA}$. Similar behavior has also been observed for many other 2D Au clusters in a size range from 3 to $5 \mathrm{~nm}$.

There are a number of possibilities that could cause the above nonmonotonic behavior. First, the strong electric field may dissociate or even evaporate atoms away from the Au cluster to result in a capacitance decrease at small electrode separation. To rule out this possibility, we can withdraw the tip away from the Au cluster through decreasing the set point tunneling current in order to subsequently reduce the field effect. As shown in Fig. 3 for a $3.5 \mathrm{~nm} \mathrm{Au}$ cluster, when the set point current first increases from 1 to $10 \mathrm{nA}$, we observe a similar behavior as in Fig. 2 with a minimum CB gap width occurring at $\sim 3 \mathrm{nA}$. When the set point current is reversed to decrease from 10 to $1 \mathrm{nA}$, the CB gap width is found to undergo a reversal change accordingly. The reversibility is quite good with only some minor changes in the spectra. Thus, the observed nonmonotonic behavior of the capacitance is not due to a change in cluster size but is intrinsic in nature.

A second possibility is that the decrease in capacitance at very small tip-cluster separation is due to a nonlinear dielectric response under strong electric field. This can exist only if the 2D Au cluster behaves nonmetallic in the field direction. Using a heuristic simple parallel plate capacitor model with a finite dielectric constant for the Au cluster in the field direction, we can write down an equivalent capacitance as $C_{2}=\varepsilon_{0} \varepsilon_{\mathrm{Au}} A /\left(d_{\mathrm{Au}}+\varepsilon_{\mathrm{Au}} d\right)$, where $\varepsilon_{0}$ is the

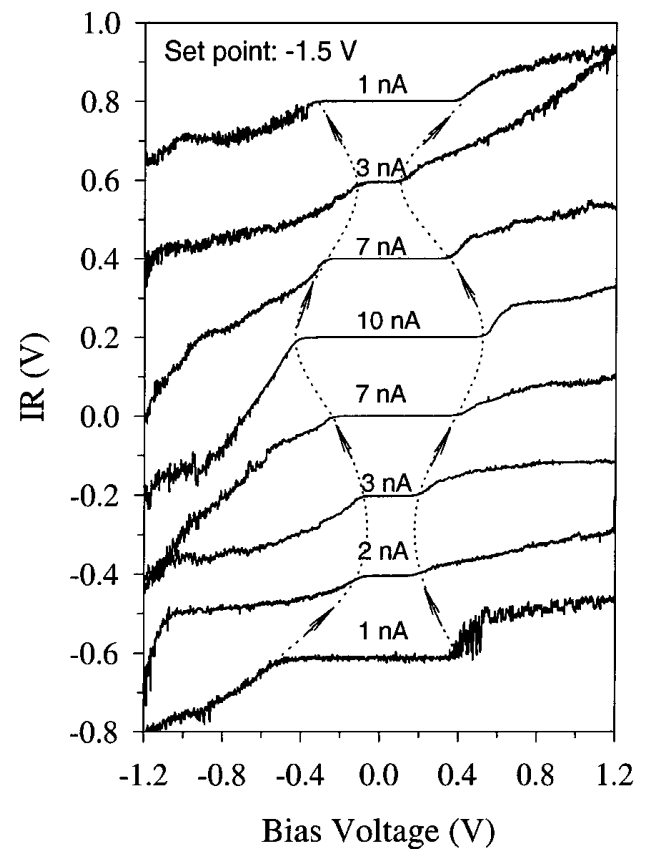

FIG. 3. $I-V$ curves of a DBTJ for a $3.5 \mathrm{~nm}$ cluster measured at $5 \mathrm{~K}$ by first increasing the set point tunneling current from 1 to $10 \mathrm{nA}$ and then decreasing from 10 to $1 \mathrm{nA}$. Here the vertical axis is rescaled by the set point tunneling resistance $R=1.5$, $0.75,0.5,0.21,0.15 \mathrm{G} \Omega$ for set point tunneling current $1,2,3$, 7 , and $10 \mathrm{nA}$, respectively. The curves are shifted vertically for clarity.

permittivity of free space, $\varepsilon_{\mathrm{Au}}, A$, and $d_{\mathrm{Au}}$ are the dielectric constant, area, and thickness of the Au cluster, respectively. Since $\varepsilon_{\mathrm{Au}}$ is no longer a constant and may decrease under strong electric field, which is $\sim 5 \times 10^{7} \mathrm{~V} / \mathrm{cm}$ and non-negligible as compared to the internal field in our case, a decrease of $C_{2}$ could be anticipated. However, whether a decrease of $\varepsilon_{\mathrm{Au}}$ by more than $50 \%$ in order to account for a decrease of $C_{2}$ by a factor of $\sim 2$ could result from the nonlinear dielectric effect remains to be investigated. Initial calculation with a local density functional formalism found only $\sim 20 \%$ decrease in the dielectric constant at an external field as strong as $3 \times 10^{8} \mathrm{~V} / \mathrm{cm}$.

A more plausible interpretation of our observation is that the capacitance at the nanoscale can no longer be described by the classical theory but requires quantum corrections. When the quantum corrections are included, the capacitance can be expressed approximately as $[2,4]$

$$
C \approx \frac{R}{\frac{1}{C_{0}}+\frac{1}{D_{\mathrm{I}}}+\frac{1}{D_{\mathrm{II}}}},
$$

where $R$ is the electron reflection probability of the nanojunction and can be approximated as $R=1-\exp (-d / l)$ with $l$ as a constant, $C_{0}$ is the classical contribution based on geometry, and $D_{i}(i=\mathrm{I}, \mathrm{II})$ is the scattering local partial DOS (LPDOS) in the electrodes $[4,16]$. Physically, Eq. (1) can be understood as follows. For a finite $D_{i}$, the electric field can penetrate into the electrode by a finite penetration depth $\lambda_{i}$ [1]. This effectively increases the 
separation between the two electrodes from $d$ to $d+\lambda_{\mathrm{I}}+$ $\lambda_{\text {II }}$ [proportional to the denominator of Eq. (1) in the case of a parallel plate capacitor] and thus reduces the capacitance $C$. For an infinitely large $D_{i}$, the electric field can be perfectly screened from the electrodes and, consequently, the capacitance $C$ is not affected. The factor of $R$ in Eq. (1) is due to the quantum tunneling, which reduces the charge storable in the electrodes from $Q$ to $R Q$ at a given voltage. Now, assuming that the $D_{i}$ takes the value of conventional DOS which is independent of the electrode separation $d$, one would predict the following behavior for the capacitance $C$. For large $d$, it is the classical contribution that dominates since $1 / C_{0}$ monotonically increases with $d$ while $1 / D_{\text {I }}+1 / D_{\text {II }}$ remains constant. As long as $1 / C_{0}>1 / D_{\mathrm{I}}+1 / D_{\mathrm{II}}$, the capacitance $C$ would increase as one decreases $d$. When $1 / C_{0} \sim 1 / D_{\text {I }}+1 / D_{\text {II }}$, saturation is reached [3]. Further decrease in $d$ results in the activation of the quantum tunneling effect. This would decrease $R$, and thus $C$. Therefore, Eq. (1) predicts a behavior for the nanojunction capacitance qualitatively in agreement with what we have observed in our experiment. Taking $l \sim(1.025 \sqrt{\phi})^{-1} \sim 0.44 \AA$ as determined by the work function of the electrodes [15] and a linearized $1 / C_{0} \sim a+\beta d$, Eq. (1) unfortunately cannot fit our data in Fig. 2(b) quantitatively by adjusting the fitting parameters $a, \beta$, and $1 / D_{\mathrm{I}}+1 / D_{\mathrm{II}}$. This may be attributed to a number of reasons: (i) the experimental data of $d$ is only an estimate; (ii) $R$ for the tip-cluster junction cannot be simply computed as if it has a parallel plate geometry as above; and (iii) most importantly, the use of constant DOS is not justified for the nanojunctions at very small electrode separation. The contribution $1 / D_{\mathrm{I}}+1 / D_{\mathrm{II}}$ in Eq. (1) is not directly related to the normal DOS but to the relevant scattering LPDOS $[4,16]$, which not only depends on the electrodes themselves but also on the geometry and the width of the barrier. This LPDOS is proportional to the dwell time of the electron in the nanojunction $[4,16]$. The dwell time describes how long the electron stays at the scattering region (barrier). Thus, when $d$ is large, the electron is mainly reflected and does not spend time at the barrier, and the LPDOS contribution comes from the normal DOS only. When $d$ is small, the dwell time is also expected to be short since the electron can go through the barrier easily. Only at intermediate $d$, the dwell time is the longest, which makes the LPDOS peaked at the intermediate electrode separation. This argument leads to a $d$ dependent $1 / D_{\text {I }}+1 / D_{\text {II }}$ and a complicated $d$-dependent capacitance $C$.

In summary, we have experimentally observed a nonclassical electrode separation variation in the capacitance of a nanojunction formed by an STM tip and a 2D gold cluster. Two possible mechanisms have been discussed. Quantitatively, the quantum effect seems to be more plau- sible since it allows a large capacitance decrease at small electrode separation. Although the above interpretations account for the qualitative features in the data, many interesting aspects of this tip-cluster nanojunction remain to be explored. These include the high voltage behavior of the $I-V$ curves, the field effect on the 2D Au clusters, and the nature of the LPDOS at the various separations. Further work to understand the observation is underway.

We thank Professor Ping Sheng for his valuable input on the understanding and presentation of the data. This work was partially supported by the National Project for the Development of Key Fundamental Sciences in China, the National Natural Science Foundation of China, the Foundation of the Chinese Ministry of Education, and the Foundation of the Chinese Academy of Science. The project was also supported by the Research Grant Council of Hong Kong through Project No. HKUST612/95P, and by the high impact area fund through the Hong Kong University of Science and Technology.

*Email address: jghou@ustc.edu.cn

${ }^{\dagger}$ To whom correspondence should be addressed. Email address: phxudong@ust.hk

[1] M. Büttiker, J. Phys. Condens. Matter 5, 9361 (1993).

[2] T. Christen and M. Büttiker, Phys. Rev. Lett. 77, 143 (1996).

[3] J. Wang, H. Guo, J.-S. Mozos, C. C. Wan, G. Taraschi, and Q.-R. Zheng, Phys. Rev. Lett. 80, 4277 (1998).

[4] X. Zhao, J. Wang, and H. Guo, Phys. Rev. B 60, 16730 (1999).

[5] M. Büttiker and A. M. Martin, Phys. Rev. B 61, 2737 (2000).

[6] Y. Katayama and D. C. Tsui, Appl. Phys. Lett. 62, 2563 (1993).

[7] S. Datta, Electronic Transport in Mesoscopic System (Cambridge University Press, Cambridge, 1995).

[8] K. Mullen, E. Ben-Jacob, R. C. Jaklevic, and Z. Schuss, Phys. Rev. B 37, 98 (1988).

[9] M. Amman, R. Wilkins, E. Ben-Jacob, P. D. Maker, and R. C. Jaklevic, Phys. Rev. B 43, 1146 (1991).

[10] A.E. Hanna and M. Tinkham, Phys. Rev. B 44, 5919 (1991).

[11] Bing Wang, Xudong Xiao, and Ping Sheng, J. Vac. Sci. Technol. B 18, 2351 (2000).

[12] Bing Wang, Xudong Xiao, Xianxiang Huang, Ping Sheng, and J. G. Hou, Appl. Phys. Lett. 77, 1179 (2000).

[13] S. D. Evans and A. Ulman, Chem. Phys. Lett. 170, 462 (1990).

[14] M. A. Rampi, O. J. A. Schueller, and G. M. Whitesides, Appl. Phys. Lett. 72, 1781 (1998).

[15] C.J. Chen, Introduction to Scanning Tunneling Microscopy (Oxford University Press, Oxford, 1993).

[16] V. Gasparian, T. Christen, and M. Büttiker, Phys. Rev. A 54, 4022 (1996). 